\title{
Predictors of Cardiac Events After Major Vascular Surgery Role of Clinical Characteristics, Dobutamine Echocardiography, and $\beta$-Blocker Therapy
}

\begin{tabular}{l}
\hline Eric Boersma, PhD \\
\hline Don Poldermans, MD, PhD \\
\hline Jeroen J. Bax, MD, PhD \\
\hline Ewout W. Steyerberg, MD, PhD \\
\hline Ian R. Thomson, MD \\
\hline Jan D. Banga, MD, PhD \\
\hline Louis L. M. van de Ven, MD, PhD \\
\hline Hero van Urk, MD, PhD \\
\hline Jos R. T. C. Roelandt, MD, PhD \\
\hline for the DECREASE Study Group \\
\hline
\end{tabular}

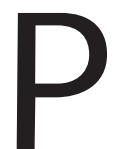
ATIENTS WITH SEVERE PERIPHeral vascular disease frequently have underlying coronary artery disease. Hence, patients undergoing major vascular surgery are at increased risk for cardiac complications during or shortly after surgery. Appropriate patient management then includes assessment of the perioperative cardiac risk, as well as strategies to reduce this risk. Several investigations demonstrated the utility of dobutamine stress echocardiography (DSE) for preoperative cardiac risk assessment. ${ }^{1-5}$ Patients with stress-induced new wall-motion abnormalities (NWMAs), a hallmark of myocardial ischemia, are at an $8 \%$ to $38 \%$ risk of cardiac death or myocardial infarction (MI) within 30 days after surgery. ${ }^{2,4,6,7}$ In contrast, patients without NWMAs have much lower complication rates: in the range of $0 \%$ to $5 \%$. There are, however, significant disadvantages associated with the routine use of DSE (or other noninvasive imaging techniques) in all vascular surgery candidates. These include the substantial costs

Context Patients who undergo major vascular surgery are at increased risk of perioperative cardiac complications. High-risk patients can be identified by clinical factors and noninvasive cardiac testing, such as dobutamine stress echocardiography (DSE); however, such noninvasive imaging techniques carry significant disadvantages. A recent study found that perioperative $\beta$-blocker therapy reduces complication rates in high-risk individuals.

Objective To examine the relationship of clinical characteristics, DSE results, $\beta$-blocker therapy, and cardiac events in patients undergoing major vascular surgery.

Design and Setting Cohort study conducted in 1996-1999 in the following 8 centers: Erasmus Medical Centre and Sint Clara Ziekenhuis, Rotterdam, Twee Steden Ziekenhuis, Tilburg, Academisch Ziekenhuis Utrecht, Utrecht, and Medisch Centrum Alkmaar, Alkmaar, the Netherlands; Ziekenhuis Middelheim, Antwerp, Belgium; and San Gerardo Hospital, Monza, Istituto di Ricovero e Cura a Carattere Scientifico, San Giovanni Rotondo, Italy.

Patients A total of 1351 consecutive patients scheduled for major vascular surgery; DSE was performed in 1097 patients (81\%), and 360 (27\%) received $\beta$-blocker therapy.

Main Outcome Measure Cardiac death or nonfatal myocardial infarction within 30 days after surgery, compared by clinical characteristics, DSE results, and $\beta$-blocker use.

Results Forty-five patients (3.3\%) had perioperative cardiac death or nonfatal myocardial infarction. In multivariable analysis, important clinical determinants of adverse outcome were age 70 years or older; current or prior angina pectoris; and prior myocardial infarction, heart failure, or cerebrovascular accident. Eighty-three percent of patients had less than 3 clinical risk factors. Among this subgroup, patients receiving $\beta$-blockers had a lower risk of cardiac complications $(0.8 \%$ [2/263]) than those not receiving $\beta$-blockers (2.3\% [20/855]), and DSE had minimal additional prognostic value. In patients with 3 or more risk factors (17\%), DSE provided additional prognostic information, for patients without stress-induced ischemia had much lower risk of events than those with stressinduced ischemia (among those receiving $\beta$-blockers, $2.0 \%$ [1/50] vs $10.6 \%[5 / 47]$ ). Moreover, patients with limited stress-induced ischemia ( $1-4$ segments) experienced fewer cardiac events (2.8\% [1/36]) than those with more extensive ischemia ( $\geq 5$ segments, 36\% [4/11]).

Conclusion The additional predictive value of DSE is limited in clinically low-risk patients receiving $\beta$-blockers. In clinical practice, DSE may be avoided in a large number of patients who can proceed safely for surgery without delay. In clinically intermediate- and high-risk patients receiving $\beta$-blockers, DSE may help identify those in whom surgery can still be performed and those in whom cardiac revascularization should be considered.

Author Affiliations are listed at the end of this article. Members of the Dutch Echocardiographic Cardiac Risk Evaluation Applying Stress Echocardiography group are listed in Poldermans D, Boersma E, Bax JJ, et al, for the Dutch Echocardiographic Cardiac Risk Evaluation Applying Stress Echocardiography Study
Group. The effect of bisoprolol on perioperative mortality and myocardial infarction in high-risk patients undergoing vascular surgery. N Engl J Med. 1999; 341:1789-1794

Corresponding Author information is at the end of the article. 
of the test, and, more importantly, the risk of delaying surgery in patients with large aortic aneurysms or critical limb ischemia. The recent Dutch Echocardiographic Cardiac Risk Evaluation Applying Stress Echocardiography (DECREASE) study demonstrated that perioperative $\beta$-adrenergic blockade with bisoprolol reduces the risk of 30-day complications in patients with NWMAs to a risk level as observed in patients without NWMAs. ${ }^{8} 9$ This finding raises the question of whether DSE is indicated in all patients scheduled for vascular surgery. Does simple perioperative administration of $\beta$-blockers reduce or eliminate the need for noninvasive preoperative cardiac testing? ${ }^{10}$ On the other hand, in some patients the cardioprotective effect of $\beta$-blockers may be insufficient to effectively reduce perioperative cardiac events. These patients may benefit from additional coronary revascularization. To address these issues we studied the relationship between clinical characteristics, DSE results, $\beta$-blockers, and adverse cardiac outcome in a large series of consecutive patients scheduled to undergo major vascular surgery.

\section{METHODS Patients}

The study population consisted of 1351 consecutive patients scheduled for elective major vascular surgery who were screened for eligibility for the DECREASE study at 8 of the following participating centers: Erasmus Medical Centre and Sint Clara Ziekenhuis, Rotterdam, Twee Steden Ziekenhuis, Tilburg, Academisch Ziekenhuis Utrecht, Utrecht, and Medisch Centrum Alkmaar, Alkmaar, the Netherlands; Ziekenhuis Middelheim, Antwerp, Belgium; and San Gerardo Hospital, Monza, Istituto di Ricovero e Cura a Carattere Scientifico, San Giovanni Rotondo, Italy. ${ }^{8}$ Per protocol, 846 patients with at least 1 of the following risk factors underwent DSE: age 70 years or older, angina, prior MI, congestive heart failure, treatment for ventricular arrhythmias, treatment for diabetes mellitus, or limited exercise capacity. Two hundred forty-five additional patients underwent DSE at the discretion of the treating physician, based on other risk factors (eg, hyperlipidemia and smoking). Thus, in total 1091 patients (81\%) underwent DSE. Perioperative $\beta$-blockers were administered to 360 patients (27\%): 301 (22\%) of the 1351 patients were treated long-term with $\beta$-blockers, and 59 (5\%) were randomized to receive $\beta$-blockers within the framework of the DECREASE study. Eight patients who had extensive resting and/or stress-induced ischemia were excluded from the DECREASE study. Four of these underwent coronary artery bypass graft surgery, and 2 of them died during this operation. The 2 surviving patients subsequently underwent uneventful vascular surgery with perioperative $\beta$-blocker administration for cardiac protection. The other 4 patients underwent vascular surgery without prior myocardial revascularization and received $\beta$-blockers for cardiac protection. None of the other nonDECREASE patients received $\beta$-blockers for cardiac protection.

\section{Dobutamine Stress Echocardiography}

Dobutamine stress echocardiography was performed according to a standard protocol. ${ }^{11}$ The left ventricle was divided into 16 segments and wall motion was scored on a 5-point ordinal scale (1, normal wall motion; 2 , mild hypokinesis; 3 , severe hypokinesis; 4 , akinesis; and 5, dyskinesis). The results of DSE were considered positive if new wall-motion abnormalities (NWMAs) occurred (ie, if wall motion in any segment worsened by $\geq 1$ more grades during the test, with the exception of akinesis becoming dyskinesis). The extent and location of ischemia were evaluated and a wallmotion index was calculated, both at rest and during peak stress.

\section{End Point Definition}

The study end point was a composite of cardiac death or nonfatal MI (MI) occurring during the period from screening until 30 days after surgery. An ad- verse events committee adjudicated all end points. Deaths were considered to be cardiac related unless there was explicit evidence for a noncardiac cause. Myocardial infarction was defined by either a serum creatine kinase level of more than $110 \mathrm{U} / \mathrm{L}$ with a myoglobin isoenzyme fraction of more than $10 \%$, or by new $\mathrm{Q}$ waves faster than 30 milliseconds in duration on the 12-lead electrocardiogram.

\section{Data Analysis}

Univariable and multivariable logistic regression analyses were applied to evaluate the relations between a limited number of baseline clinical characteristics, DSE results, $\beta$-blocker therapy, and the composite end points as outlined above. All variables that reached a $P$ value $<.50$ in univariable analysis entered the multivariable stage. Multivariable models were constructed by backward deletion of the least significant characteristics, applying the Akaike optimal information criterion. ${ }^{12,13}$ Special attention was paid to the extent to which DSE results and $\beta$-blocker therapy contributed to the prognostic information obtained from clinical characteristics alone. Odds ratios (ORs) and corresponding 95\% confidence intervals (CIs) are reported.

Despite the fact that the population consisted of patients undergoing highrisk surgery, the number of outcome events appeared to be limited. Therefore, there was a serious concern that model overfitting would occur. To overcome this, we limited the number of candidate clinical variables to be included in the model, whereby, particularly, the results of prior risk modeling analyses in surgical patients were considered. ${ }^{14-17}$ Furthermore, we applied a clinical risk model that was developed elsewhere to our data set, and collapsed the clinical risk assessment into 1 index variable. Subsequently, the prognostic value of this risk index and the additional and additive prognostic value of DSE results and $\beta$-blocker therapy were analyzed by logistic regression analyses. We chose the risk index that was recently developed by Lee 
and colleagues ${ }^{17}$ in a data set of 4315 patients undergoing major noncardiac surgery, including 898 patients undergoing vascular surgery. To compose the Lee risk index, 1 point is assigned to each of the following characteristics: high-risk type of surgery, known ischemic heart disease, a history of congestive heart failure, a history of cerebrovascular disease, diabetes mellitus, and renal failure.

The performance of the risk models was determined by the cardiac index, which indicates how well a model rankorders patients with respect to their outcomes; the cardiac index ranges from 0.5 (not predictive at all) to 1.0 (optimal performance). ${ }^{18}$ In addition, the Hosmer-Lemeshow statistic for goodness of fit is presented. The predictive accuracy of the models was further evaluated by bootstrapping techniques. ${ }^{19}$ One hundred bootstrap samples were drawn from the original data set (with replacement) and the full modeling process, including the stepwise selection, was redone in every bootstrap sample. The models developed in the bootstrap samples were subsequently tested in the original data set. This process provides a factor to correct for a possible overoptimism of the cardiac index. ${ }^{20}$

Based on the results obtained by the described modeling strategies, a risk score was developed to estimate an individual patient's risk of perioperative cardiac death or MI. Furthermore, a simple decision-tree is constructed to help the physician decide in which patients to refer for noninvasive perioperative cardiac testing.

\section{RESULTS}

The primary patient characteristics are described in TABLE 1. Obviously, patients receiving $\beta$-blockers during surgery had a risk profile that was worse than that of patients not taking such medication because they had higher rate of hypertension, ventricular arrhythmias, and history of coronary disease. Furthermore, patients receiving $\beta$-blockers more frequently used other cardiac medications than those who were not receiving $\beta$-blockers. There were 45 perioperative cardiac complications (3.3\%): 31 patients had cardiac death and another 14 nonfatal MI.

\section{Univariable Models}

In univariable analysis, a history of heart failure was the most significant determinant of adverse cardiac outcome among the clinical variables examined (TABLE 2). The subgroup of patients with a history of heart failure (5\% of the population) had a more than 5-fold increase in the risk of perioperative cardiac death or MI compared with those without such a history. Other important univariable determinants of perioperative cardiac complications were a history of MI, prior cerebrovascular accident (CVA), current or prior angina pectoris, and age 70 years and older. Patients taking cardiac medications had higher event rates than patients not taking such medication; statistical significance was observed for nitrates and angiotensin-converting enzyme (ACE) inhibitors. There was no relationship between the type of surgery and the composite end point.

Patients who did not undergo DSE (ie, patients without clinical cardiac risk factors) and those without NWMAs during DSE had a significantly lower cardiac death or MI rate than patients with NWMAs during DSE $(0.4 \%$ and $1.6 \%$ vs $13.5 \%$, respectively $(P<.001$; TABLE 3). Thus, NWMAs were strongly predictive of adverse perioperative cardiac outcome. Moreover, the extent of stress-induced ischemia also provided important prognostic information, as the event rates ranged from $10.8 \%$ in those with NWMAs in 1 to 4 segments

Table 1. Characteristics of the Population

\begin{tabular}{|c|c|c|c|}
\hline Characteristics & $\begin{array}{l}\text { Patients Who } \\
\text { Used } \beta \text {-Blockers* }\end{array}$ & $\begin{array}{l}\text { Patients Who Did } \\
\text { Not Use } \beta \text {-Blockers }\end{array}$ & $P$ Value \\
\hline Patients, No. & $360(100)$ & $991(100)$ & \\
\hline \multicolumn{4}{|l|}{ Demographics } \\
\hline Age $\geq 70$ years & $156(43)$ & $425(43)$ & .9 \\
\hline Men & $280(78)$ & $774(78)$ & .94 \\
\hline \multicolumn{4}{|l|}{ History } \\
\hline Diabetes mellitus & $56(16)$ & $129(13)$ & .28 \\
\hline Ventricular arrhythmias & $20(6)$ & $26(3)$ & .01 \\
\hline Current stable angina or prior angina & $158(44)$ & $176(18)$ & $<.001$ \\
\hline Prior myocardial infarction & $210(59)$ & $281(29)$ & $<.001$ \\
\hline Congestive heart failure & $27(8)$ & $44(5)$ & .04 \\
\hline $\begin{array}{l}\text { Percutaneous transluminal } \\
\text { coronary angioplasty }\end{array}$ & $33(9)$ & $26(3)$ & $<.001$ \\
\hline Coronary artery bypass graft surgery & $66(19)$ & $100(10)$ & $<.001$ \\
\hline Prior cerebrovascular accident & $39(11)$ & $78(8)$ & .1 \\
\hline Pulmonary disease & $49(14)$ & $122(13)$ & .58 \\
\hline Renal failure & $19(5)$ & $36(4)$ & .21 \\
\hline Aortic valve stenosis & $14(4)$ & $25(3)$ & .2 \\
\hline $\begin{array}{l}\text { Long-term cardiac medication } \\
\text { Aspirin }\end{array}$ & $134(38)$ & $274(29)$ & $<.01$ \\
\hline Nitrates & $94(27)$ & $113(12)$ & $<.001$ \\
\hline Calcium antagonists & $135(38)$ & $200(21)$ & $<.001$ \\
\hline $\begin{array}{l}\text { Angiotensin-converting } \\
\text { enzyme inhibitors }\end{array}$ & $127(36)$ & $267(28)$ & $<.01$ \\
\hline Lipid-lowering agents & $120(34)$ & $166(18)$ & $<.001$ \\
\hline \multicolumn{4}{|l|}{ Scheduled type of surgery } \\
\hline Tube prosthesis & $69(19)$ & $179(18)$ & \multirow[t]{2}{*}{.83} \\
\hline Aortic-femoral bypass graft & $166(46)$ & $474(48)$ & \\
\hline \multicolumn{4}{|c|}{$\begin{array}{l}\text { * } 3 \text {-blocker therapy included long-term users }(n=301) \text { and patients randomized to bisoprolol as part of the Dutch Echo- } \\
\text { cardiographic Cardiac Risk Evaluation Applying Stress Echocardiography (DECREASE) study }(n=59) \text {. All data are } \\
\text { presented as number (percentage) unless otherwise indicated. } \\
\text { †Fisher exact 2-sided test. }\end{array}$} \\
\hline
\end{tabular}

* $\beta$-blocker therapy included long-term users $(n=301)$ and patients randomized to bisoprolol as part of the Dutch Echocardiographic Cardiac Risk Evaluation Applying Stress Echocardiography (DECREASE) study ( $n=59)$. All data are presented as number (percentage) unless otherwise indicated. †Fisher exact 2-sided test. 
Table 2. Univariable Relation Between Clinical Baseline Characteristics and Perioperative Cardiac Death or Myocardial Infarction ( $\mathrm{N}=1351)^{*}$

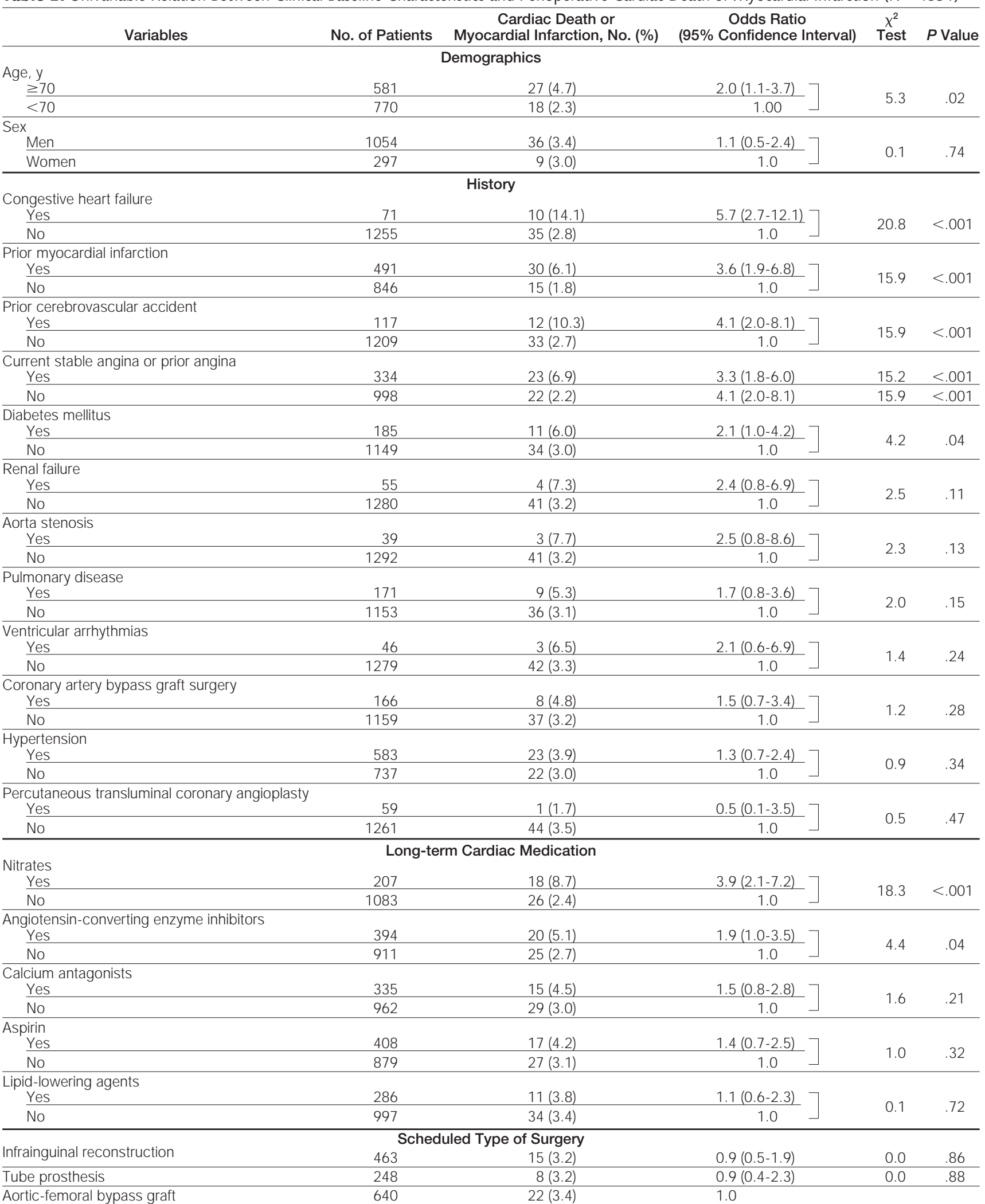

*Numbers may not add to 1351 due to missing data.

1868 JAMA, April 11, 2001—Vol 285, No. 14 (Reprinted) 
to $23.9 \%$ in patients with NWMAs in 5 or more segments. The echocardiogram at rest also provided prognostic information. Patients with 5 or more abnormal segments had a 4- to 6-fold increased risk of cardiac complications compared with those with a normal or slightly aberrant (1-4 abnormal segments) wall motion at rest.

Despite their overall risk profile being worse (Table 1), patients receiving $\beta$-blockers during surgery had a significantly reduced risk of cardiac death or MI compared with those not taking such medication. Among the 254 patients who did not undergo DSE, no perioperative cardiac complications were observed in the $8.7 \%$ of patients receiving $\beta$-blockers, whereas there was 1 event $(0.4 \%)$ in the remaining patients. In the 875 patients without stress-induced NWMAs, 22\% were receiving $\beta$-blockers. One cardiac complication $(0.5 \%)$ occurred in this group, and there were 13 $(1.9 \%)$ in those not receiving $\beta$-blockers. Finally, in the 222 patients with NWMAs, $67 \%$ of those receiving $\beta$-blockers with $4.7 \%$ having a perioperative cardiac event vs $31.5 \%$ among those not receiving $\beta$-blockers. There was no evidence of a differential effect of $\beta$-blocker therapy in these patient cat- egories (homogeneity test for ORs, $P=.69$ ), so that the crude OR is best estimated by the method of MantelHaenszel test (0.1; 95\% CI, 0.1-0.3).

\section{Multivariable Models}

Many of the univariably significant clinical determinants of cardiac outcome remained important in the multivariable analysis (TABLE 4). After correcting for other determinants, prior CVA showed the strongest relationship with perioperative cardiac complications. A history of heart failure and prior MI were the next strongest clinical predictors. Angina and age 70 years or more were also important. After correcting for differences in clinical characteristics, patients receiving $\beta$-blockers were still at significantly lower risk for the composite end point than those who were not (adjusted OR, 0.3; 95\% CI, 0.1-0.7).

When clinical data were combined with DSE results, advanced age, angina pectoris, prior MI, and prior heart failure lost most of their predictive power with respect to the composite end point. In fact, DSE results (especially the presence or absence of NWMAs) were the most important determinants of perioperative cardiac outcome. In connection with both clinical data and DSE results, $\beta$-blocker therapy was again associated with a significantly reduced risk of the composite end point. The protective effect of $\beta$-blocker therapy was observed in long-term users (OR, 0.1; 95\% CI, 0.0$0.3)$ as well as in patients who received bisoprolol as part of the DECREASE study (OR, 0.1; 95\% CI, 0.0-0.4).

\section{Application of the Lee Risk Index}

In all, 611 patients (45\%) had a Lee risk index of 1, 509 (38\%) had an index of 2 , and 231 (17\%) had an index of 3 or more points (note that all patients underwent high-risk surgery, and thus had a risk index $\geq 1$ points). The incidence of the composite end point in these patients was $1.3 \%, 3.1 \%$, and $9.1 \%$ $(P<.001)$. Regression analysis revealed a crude OR of 2.3 for the composite end point associated with a 1 -point increase in the risk index (95\% CI, 1.8-3.1). Multivariable analyses again demonstrated the additional and additive prognostic value of DSE results and $\beta$-blocker therapy (TABLE 5).

\section{Predictive Accuracy}

The cardiac index for the composite end point model based on clinical characteristics only was 0.78 , reflecting good ability to discriminate between patients who

Table 3. Univariable Relation Between Dobutamine Stress Echocardiography Results and Perioperative Cardiac Death or Myocardial Infarction*

\begin{tabular}{|c|c|c|c|c|c|}
\hline Variables & No. of Patients & $\begin{array}{l}\text { Cardiac Death or } \\
\text { Myocardial Infarction, No. (\%) }\end{array}$ & $\begin{array}{l}\text { Odds Ratio } \\
\text { (95\% Confidence Interval) }\end{array}$ & $\chi^{2}$ & $P$ Value \\
\hline $\begin{array}{l}\text { DSE summary } \\
\text { New wall-motion abnormalities }\end{array}$ & 222 & $30(13.5)$ & $39.5(5.3-292)$ & 13.0 & $<.001$ \\
\hline No new wall-motion abnormalities & 875 & $14(1.6)$ & $4.1(0.5-8.1)$ & \multirow{2}{*}{1.9} & \multirow{2}{*}{.17} \\
\hline No DSE & 254 & $1(0.4)$ & 1.0 & & \\
\hline $\begin{array}{l}\text { Segments with new wall-motion abnormalities, No.† } \\
\geq 5\end{array}$ & 46 & $11(23.9)$ & $19.3(8.2-45.6)$ & 45.7 & $<.001$ \\
\hline $1-4$ & 176 & $19(10.8)$ & $7.4(3.7-15.2)$ & \multirow{2}{*}{30.6} & \multirow{2}{*}{$<.001$} \\
\hline 0 & 875 & $14(1.6)$ & 1.0 & & \\
\hline $\begin{array}{l}\text { Abnormal segments at rest, No. } † \\
\quad \geq 5\end{array}$ & 305 & $29(9.5)$ & $5.8(2.8-12.2)$ & 22.3 & $<.001$ \\
\hline $1-4$ & 226 & $5(2.2)$ & $1.3(0.4-3.7)$ & \multirow{2}{*}{0.2} & \multirow{2}{*}{.68} \\
\hline 0 & 566 & $10(1.8)$ & 1.0 & & \\
\hline $\begin{array}{l}\text { ST-segment changes during testt } \\
\text { Yes }\end{array}$ & 233 & $20(8.6)$ & $3.7(1.9-6.9)$ & \multirow{2}{*}{16.2} & \multirow{2}{*}{$<.001$} \\
\hline No & 839 & $21(2.5)$ & 1.0 & & \\
\hline $\begin{array}{l}\text { Angina during testt } \\
\text { Yes }\end{array}$ & 100 & $10(10.0)$ & $3.3(1.6-7.0)$ & \multirow{2}{*}{10.0} & \multirow{2}{*}{.002} \\
\hline No & 986 & $32(3.2)$ & 1.0 & & \\
\hline
\end{tabular}


did and did not have a life-threatening cardiac complication. The associated goodness of fit of the $\chi_{7}^{2}$ statistic was 2.1 $(P=.95)$. After correction for overoptimism the cardiac index was 0.72 , still reflecting satisfactory performance. The multivariable model that combined clinical data with DSE results had considerably better discriminating power with a cardiac index of 0.87 (goodness of fit $\chi_{6}^{2}=7.6, P=.27$; optimism-corrected car- diac index, 0.82). The cardiac index connected with the Lee risk-index-alone model was 0.71 (goodness of fit $\chi_{6}^{2}, 7.0$; $P=.32$ ) and improved to 0.87 by adding DSE results and information regarding $\beta$-blocker therapy.

\section{Risk Classification Model}

Based on the results described above, a simple scheme was developed to estimate a patient's risk of perioperative car-

Table 4. Multivariable Models to Predict Perioperative Cardiac Death or Myocardial Infarction*

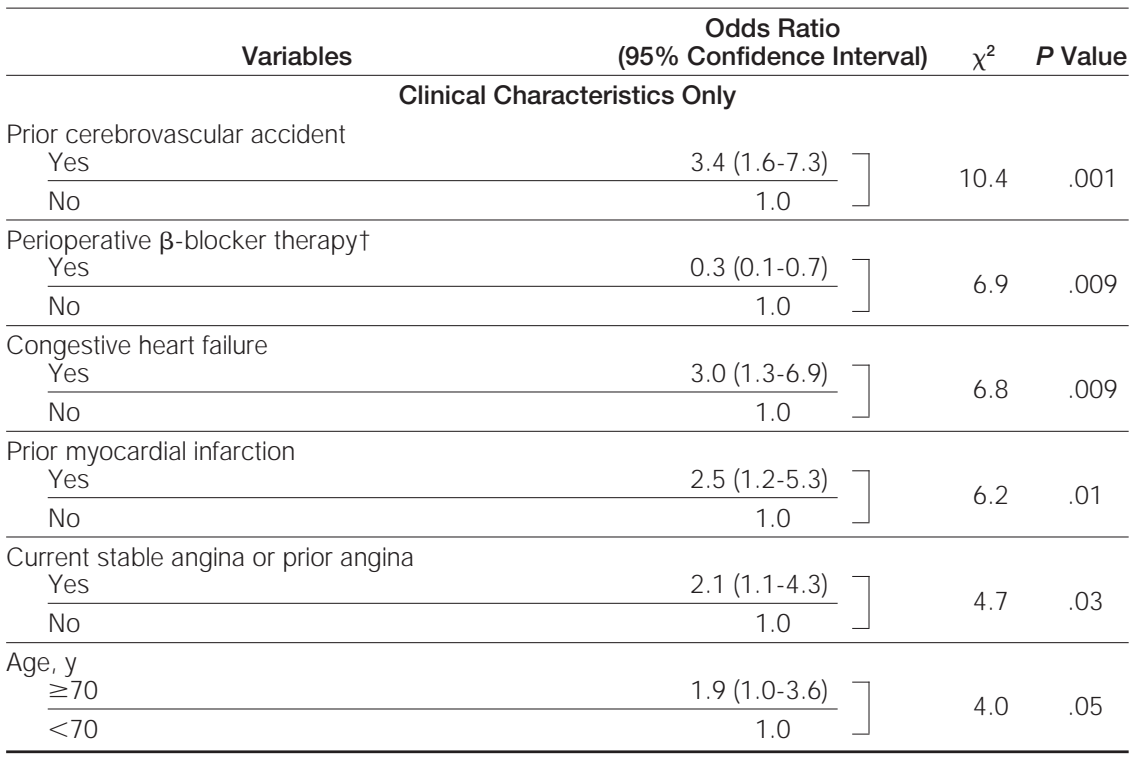

Combination of Clinical Characteristics and Results of Dobutamine Stress Echocardiography

Segments with new wall-motion abnormalities, No.

$\left.\begin{array}{lcc}\geq 5 & 16.7(6.0-47.0) & 39.3<.001 \\ \hline 1-4 & 13.9(6.1-31.7) \\ \hline 0 & 1.0\end{array}\right] 28.5<.001$

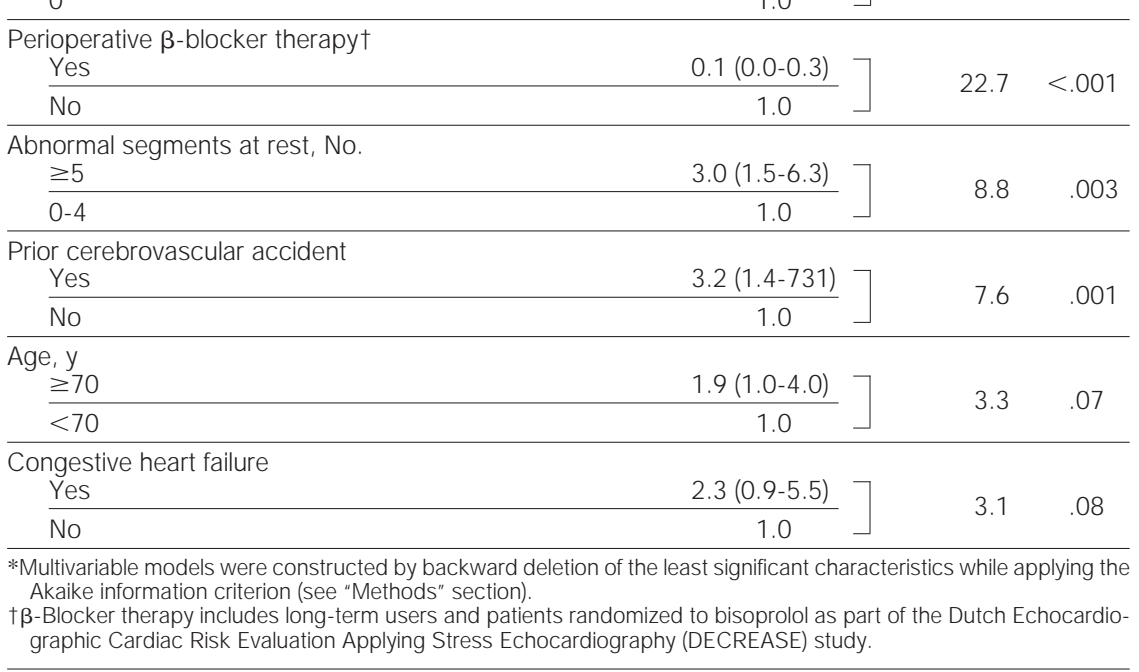

diac complications (FIGURE 1). A clinical risk score can be determined on the basis of the patient's age and clinical history. If this risk score is in the range of 0 to 2 points ( $83 \%$ of the patients belonged to this category) and the $\beta$-blockers are administered perioperatively, the estimated cardiac complication rate is relatively low $(<2 \%)$, irrespective of the DSE result. The estimated risk of cardiac complications is also low in patients with a risk score of 3 or more points without NWMAs, provided $\beta$-blockers will be applied. Patients with a risk score of 3 or more and NWMAs were at a considerable cardiac risk $(>6 \%)$, despite $\beta$-blocker therapy.

\section{COMMENT}

Consistent with other studies, ${ }^{14-17}$ our analysis of 1351 patients undergoing high-risk noncardiac vascular surgery demonstrated that advanced age, current or prior angina, and a history of cardiac or cerebral events are the most important clinical determinants of perioperative cardiac death or MI. Apart from clinical data, DSE results were highly predictive of adverse cardiac outcome, which also confirms other investigations. ${ }^{1}$ Patients receiving $\beta$-blockers had significantly lower risk than those not receiving them. It should be emphasized that patients receiving $\beta$-blockers had a considerably worse overall risk profile than those not receiving them, which makes this result even more convincing. The additional and additive prognostic value of DSE results and $\beta$-blocker therapy was confirmed in the analysis that applied the previously developed Lee risk index. On the basis of a risk score composed of a weighted sum of the prognostic clinical characteristics, a large group (83\%) of low-risk patients with a score of less than 3 could be defined. In this group, the estimated risk of cardiac complications is less than $1 \%$, regardless of DSE results, as long as patients are receiving $\beta$-blockers. In the remaining patients, those without stress-induced ischemia also had a low estimated cardiac risk in the presence of perioperative $\beta$-blocker therapy. 
Univariable analyses showed that patients with diabetes mellitus, pulmonary disease, prior ventricular arrhythmias, or aortic valvular stenosis are at increased risk of surgical complications. However, in contrast to earlier studies, ${ }^{16,21}$ these factors were not independent predictors in our multivariable analyses. This finding can be reflect a changing patient population or improved perioperative management although it may also be a matter of (lack of) power. Additionally, it should be emphasized that cardiac death or MI during complex surgery is most likely to occur in patients with stressinducible cardiac ischemia. Indeed, the occurrence of NWMAs during DSE was a major determinant of adverse outcome. Because a stress-induced ische-
Table 5. Model for the Prediction of Cardiac Death or Myocardial Infarction by the Lee Risk Index, Dobutamine Stress Echocardiography Results, and Perioperative $\beta$-Blocker Therapy

\begin{tabular}{|c|c|c|c|}
\hline Variables & $\begin{array}{c}\text { Odds Ratio } \\
\text { (95\% Confidence Interval) }\end{array}$ & $\chi^{2}$ & $P$ Value \\
\hline $\begin{array}{l}\text { Segments with new wall-motion } \\
\text { abnormalities, No. } \\
\geq 5\end{array}$ & $11.6(4.4-30.5)$ & 24.8 & $<.001$ \\
\hline $1-4$ & $6.7(3.1-14.5)$ & 23.1 & $<.001$ \\
\hline$\overline{0}$ & 1.0 & & \\
\hline $\begin{array}{l}\text { Perioperative } \beta \text {-blocker therapy* } \\
\text { Yes }\end{array}$ & $0.2(0.1-0.6)$ & 10.4 & .001 \\
\hline No & 1.0 & & \\
\hline $\begin{array}{l}\text { Abnormal segments at rest, No. } \\
\quad \geq 5\end{array}$ & $2.7(1.3-5.5)$ & 7.1 & .008 \\
\hline$\overline{0-4}$ & 1.0 & & \\
\hline $\begin{array}{l}\text { Lee risk index† } \\
\text { 1-Point increase }\end{array}$ & $1.6(1.1-2.2)$ & 6.5 & .01 \\
\hline
\end{tabular}

Figure 1. Estimate of the Perioperative Risk of Cardiac Death or Myocardial Infarction

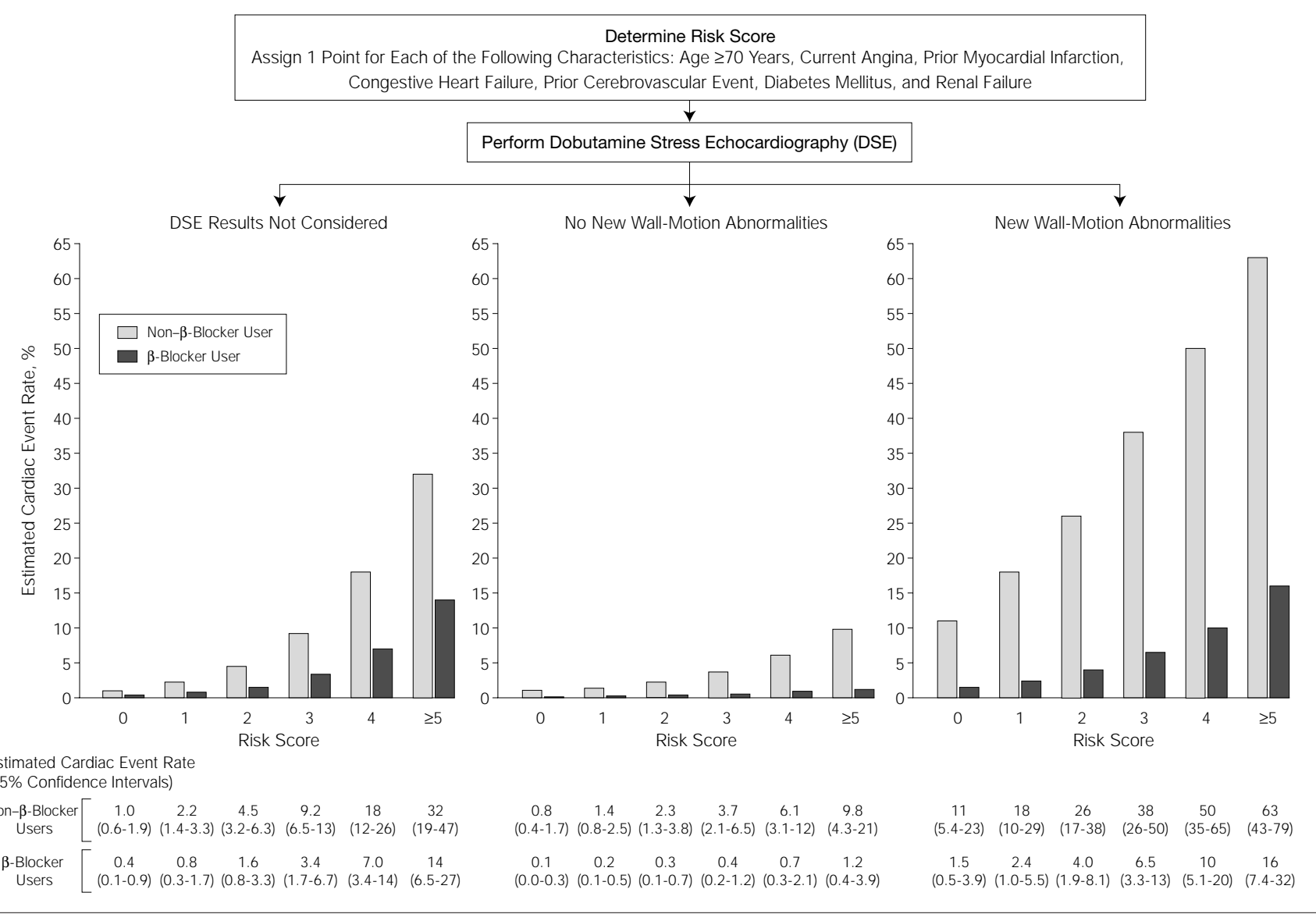

Results are based on clinical characteristics and results of DSE in patients who received $\beta$-blockers and those who did not receive $\beta$-blockers. Results reflect predictions based on logistic regression analyses. 
Figure 2. Perioperative Cardiac Risk of Death or Myocardial Infarction as Observed in Subpopulations

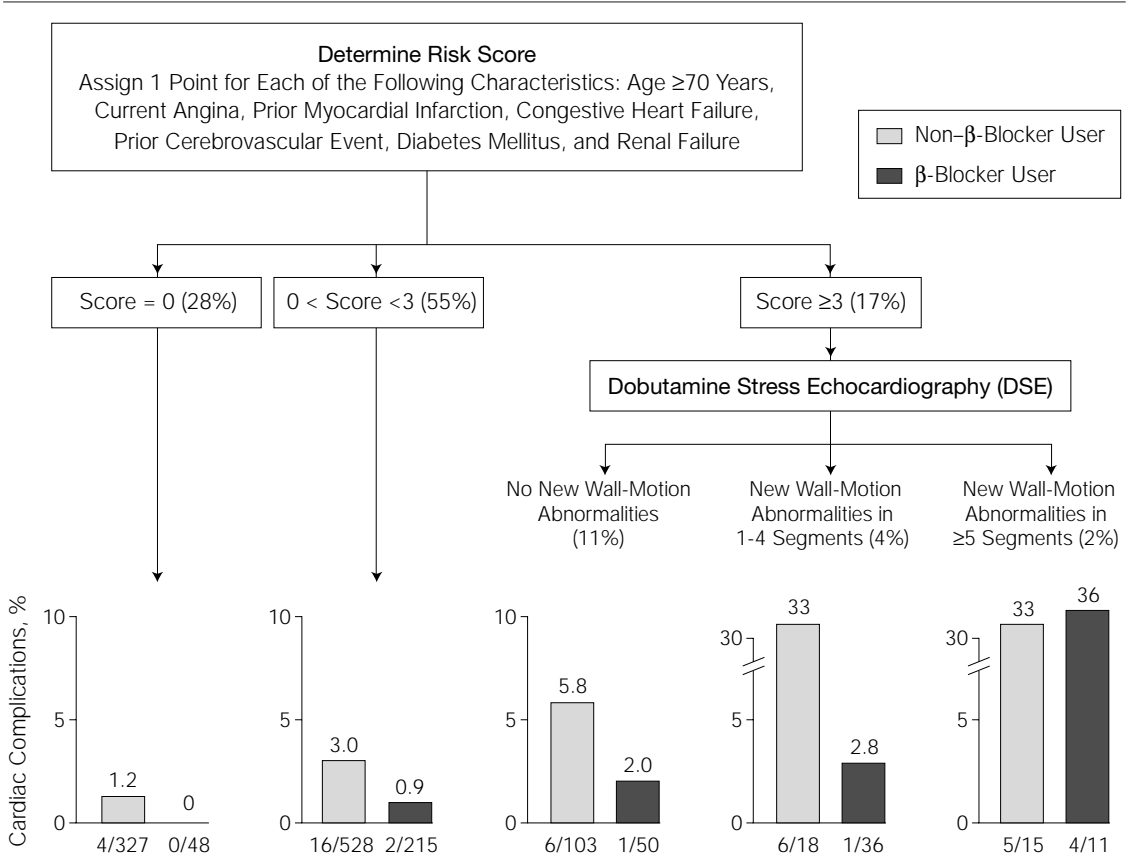

Results are according to the clinical risk score, dobutamine stress echocardiography, and receipt of $\beta$-blockers during surgery. Percentages in parentheses represent the number of patients in the target category as a proportion of the total number of 1351 patients. Numbers underneath the bars represent the actual number of events/patients in the specific category.

mia was not more common in patients with diabetes mellitus, pulmonary disease, prior ventricular arrhythmias, or aortic stenosis ( 78 NWMAs of 352 cases [22\%]) than in patients without such characteristic (144 NWMAs of 745 cases $[19 \%] ; P=.28)$, these characteristics are likely not strong determinants of a predisposition to ischemia. In contrast, patients with prior MI, prior heart failure, or prior CVA were more likely to have stress-induced ischemia than other patients (150 NWMAs of 545 cases [28\%] vs 72 NWMAs of 552 cases [13\%]; $P<.001)$. Still, factors such as diabetes mellitus, may be predictive of long-term complications. Diabetes mellitus (and renal failure) were therefore still incorporated in the clinical risk index (Figure 1).

\section{Clinical Implications}

FIGURE 2 may help to understand how results can be translated into clinical practice. The perioperative cardiac event rate was low ( $\geq 1 \%$ ) in patients with a clinical risk score of less than 3 points and who were receiving $\beta$-blockers. It seems therefore appropriate to omit DSE (and other noninvasive cardiac testing) in this large ( $>80 \%)$ group of patients and to proceed expeditiously with surgery under protection by $\beta$-blocker therapy. Dobutamine stress echocardiography is useful to further riskstratify patients with a clinical risk score of 3 or more points. If protected by perioperative $\beta$-blockade, patients without stress-induced ischemia still had a low complication rate (2\%) and are also candidates for prompt surgery. Patients with a risk score of 3 or more points and NWMAs (approximately $6 \%$ of the population) had a considerable complication rate despite the $\beta$-blocker therapy. Our data suggest that the proposed treatment policy in these patients may depend on the extent of stress-induced ischemia. Although the numbers of patients and events are relatively small in the specific subgroups, patients with NWMAs in 1 to 4 segments were properly protected by $\beta$-blockers. In patients with more extensive ischemia, however, $\beta$-blockers failed to reduce the rate of perioperative cardiac complications. Cardiac catheterization and subsequent myocardial revascularization should be considered in these patients.

The prescription of $\beta$-blockers may delay surgery; so far, no study has indicated what the optimal run-in period of this drug is in this setting. Therefore, it can be questioned whether such therapy is really necessary in patients at very low risk. In the group of patients with a risk score of 0 points, $1.2 \%$ perioperative complications were observed in those without $\beta$-blocker therapy (Figure 2). This complication rate seems sufficiently low to refrain from administering medication indeed and opt for surgery without delay. Another issue is that $\beta$-blocker therapy may be contraindicated, especially in patients with reactive airway diseases, such as severe asthma or chronic obstructive pulmonary disease with a reactive component. It should be noticed that these patients are rare: there were no such cases in our data set. Still, if $\beta$-blocker therapy is contraindicated, the use of calcium antagonists with a negative chronotropic effect may be considered. The recent Incomplete Infarction Trial of European Research Collaborators Evaluating Prognosis postThrombolysis (INTERCEPT) study of post-MI patients reported fewer cardiac events in patients randomized to such a drug compared with placebo. ${ }^{22}$

\section{Study Limitations}

Because of its retrospective nature, our analysis has limitations, which should be considered when interpreting the results. The risk-stratification and modification scheme using a clinical risk score, DSE, and $\beta$-blocker therapy was developed after events had occurred. Furthermore, only some patients (those participating in the DECREASE study) were randomized to receive either perioperative $\beta$-blockers or standard care. Patient characteristics may have played 
an important role in the decision to administer $\beta$-blockers to patients who did not participate in the DECREASE study and could potentially bias the results. Importantly, we found no difference in the cardioprotective effect of $\beta$-blockers between patients who were randomized within the framework of the DECREASE study and those whose receipt of $\beta$-blockers was chronic. This suggests that the utility of perioperative $\beta$-adrenergic blockade extends beyond the small subset of high-risk patients who participated in the DECREASE study. Finally, the observed event rates were relatively low compared with previous investigations. It should be appreciated that our results were obtained in selected, highvolume hospitals (3 of the participat- ing hospitals were university hospitals) and that cardiac event rates might differ in other centers.

\section{Conclusions}

Dobutamine stress echocardiography effectively identifies patients at risk for perioperative cardiac events. Besides, our data showed that the additional predictive value of DSE is limited in clinically low-risk patients receiving $\beta$-blockers. This observation may have important clinical implications: in a majority of patients, additional testing by DSE can be avoided and patients can proceed safely for surgery without delay. In a smaller group of clinically intermediate- and high-risk patients, DSE may help to identify patients in whom surgery can still be performed while receiving $\beta$-block- ers and those in whom cardiac revascularization should be considered.

Author Affiliations: University Hospital Rotterdam, Rotterdam (Drs Boersma, Poldermans, Steyerberg, van de Ven, van Urk, and Roelandt), the University Hospital Leiden, Leiden (Dr Bax), and University Hospital Utrecht, Utrecht (Dr Banga), the Netherlands; and University of Manitoba, Winnipeg ( $\mathrm{Dr}$ Thomson).

Corresponding Author and Reprints: Don Poldermans, MD, PhD, University Hospital Rotterdam, Department of Surgery, Room H921, Dr Molewaterplein 40, 3015 GD Rotterdam, the Netherlands (e-mail: poldermans@hlkd.azr.nl).

Author Contributions: Study concept and design: Boersma, Poldermans, Roelandt.

Acquisition of data: Boersma, Poldermans.

Analysis and interpretation of data: Boersma, Poldermans, Bax, Steyerberg, Thomson, Banga, van de Ven, van Urk.

Drafting of the manuscript: Boersma, Poldermans.

Critical revision of the manuscript for important intellectual content: Bax, Thomson, Banga, van de Ven, van Urk, Roelandt.

Statistical expertise: Boersma, Steyerberg.

Study supervision: Roelandt.

\section{REFERENCES}

1. Poldermans D, Fioretti PM, Forster T, et al. Dobutamine stress echocardiography for assessment of perioperative cardiac risk in patients undergoing major vascular surgery. Circulation. 1993;87:1506-1512.

2. Poldermans $D$, Arnese $M$, Fioretti PM, et al. Improved cardiac risk stratification in major vascular surgery with dobutamine-atropine stress echocardiography. J Am Coll Cardiol. 1995:26:648-653.

3. Pellikka PA, Roger VL, Oh JK, Seward JB, Tajik AJ. Safety of performing dobutamine stress echocardiography in patients with abdominal aortic aneurysm $>$ or $=4 \mathrm{~cm}$ in diameter. Am J Cardiol. 1996;77:413-416. 4. Davila-Roman VG, Waggoner AD, Sicard GA, Geltman EM, Schechtman KB, Perez JE. Dobutamine stress echocardiography predicts surgical outcome in patients with an aortic aneurysm and peripheral vascular disease. J Am Coll Cardiol. 1993;21:957-963. 5. Poldermans D, Bax JJ, Thomson IR, et al. Role of dobutamine stress echocardiography for preoperative cardiac risk assessment before major vascular surgery. Echocardiography. 2000;17:79-91.

6. Lalka SG, Sawada SG, Dalsing MC, et al. Dobutamine stress echocardiography as a predictor of cardiac events associated with aortic surgery. $J$ Vasc Surg. 1992;15:831-840.

7. Langan EM, Youkey JR, Franklin DP, Elmore JR, Costello JM, Nassef LA. Dobutamine stress echocardiography for cardiac risk assessment before aortic surgery. J Vasc Surg. 1993;18:905-911.
8. Poldermans D, Boersma E, Bax JJ, et al. The effect of bisoprolol on perioperative mortality and myocardial infarction in high-risk patients undergoing vascular surgery. N Engl J Med. 1999;341:1789-1794.

9. Lee TH. Reducing risk in noncardiac surgery. N Eng/ J Med. 1999;341:1838-1840.

10. Mayet C, Wolfe J, Foale R. Cardiac risk stratification [letter]. Lancet. 2000;355:500.

11. McNeill AJ, Fioretti PM, el-Said SM, Salustri A, Forster T, Roelandt JR. Enhanced sensitivity for detection of coronary artery disease by addition of atropine to dobutamine stress echocardiography. Am J Cardiol. 1992;70:41-46

12. Akaike H. Information theory as an extension of the maximum likelihood principle. In: Petrov BN, Csaki $\mathrm{F}$, eds. Second International Symposium on Information Theory. Budapest, Hungary: Akademia Kiado; 1973:267-281.

13. Boersma E, Pieper KS, Steyerberg EW, et al. Predictors of outcome in patients with acute coronary syndromes without persistent ST-segment elevation: results from an international trial of 9461 patients. Circulation. 2000;101:2557-2567.

14. Mangano DT, Goldman L. Preoperative assessment of patients with known or suspected coronary disease. N Eng/ J Med. 1995;333:1750-1756.

15. Eagle $\mathrm{KA}$, Brundage $\mathrm{BH}$, Chaitman $\mathrm{BR}$, et al, for the Committee on Perioperative Cardiovascular Evaluation for Noncardiac Surgery. Guidelines for perioperative cardiovascular evaluation for noncardiac surgery: Report of the American College of Cardiology/American Heart Association Task Force on Practice Guidelines. Circulation. 1996;93:12781317

16. L'Italien GJ, Paul SD, Hendel RC, et al. Development and validation of a Bayesian model for perioperative cardiac risk assessment in a cohort of 1,081 vascular surgical candidates. J Am Coll Cardiol. 1996; 27:779-786.

17. Lee TH, Marcantonio ER, Mangione CM, et al. Derivation and prospective validation of a simple index for prediction of cardiac risk of major noncardiac surgery. Circulation. 1999;100:1043-1049.

18. Kendal DG. Rank Correlation Methods. London, England: Charles Griffin; 1962.

19. Gong G. Cross-validation, the jackknife, and the bootstrap: excess error estimation in forward logistic regression. J Am Stat Assoc. 1986;81:108-113.

20. Van Houwelingen JC, Le Cessie S. Predictive value of statistical models. Stat Med. 1990;9:1303-1325.

21. Mangano DT. Perioperative cardiac morbidity. Anesthesiology. 1990;72:153-184.

22. Boden WE, van Gilst WH, Scheldewaert RG, et al for the Incomplete Infarction Trial of European Research Collaborators Evaluating Prognosis post-Thrombolysis (INTERCEPT). Diltiazem in acute myocardial infarction treated with thrombolytic agents: a randomised placebocontrolled trial. Lancet. 2000:355:1751-1756. 